\title{
Children of a lesser god: From radiocardiogram to CZT GSPECT
}

\author{
Assuero Giorgetti, MD, a and Paolo Marzullo, $\mathrm{MD}^{\mathrm{a}}$ \\ a Fondazione Toscana Gabriele Monasterio, Pisa, Italy
}

Received Oct 29, 2018; accepted Oct 29, 2018

doi: $10.1007 / \mathrm{s} 12350-018-01512-y$

\section{See related article, pp. 2017-2026}

We had the fortune to work in the Institute founded and directed for more than 40 years by Luigi Donato, a pioneer of nuclear medicine, among the most influential cardiologist of the past century. He loves to remember those days in the 1950s, when he, a young research fellow, drove every month from Pisa to Ciampino airport in Rome to wait the arrival of $\mathrm{Na} 24$. Then, he was used to put the box with the radioisotope into the mythical Italian car "Fiat Topolino" (i.e., "baby tiny mouse'), coming back to Pisa and projecting his research work. Professor Donato is one of the fathers of radiocardiography, a technique based on the radioisotopic application of the indicator-dilution principle, representing the first successful attempt to noninvasively obtain information on cardiopulmonary circulation $^{1}$ (Figure 1). A few years later, Folse and Braunwald described a radioisotope indicator-dilution technique to assess the 'fraction of left ventricular volume ejected per beat and ventricular enddiastolic and residual volumes' ${ }^{2}$ It is still difficult to fully understand how these studies have influenced cardiology. Successively, cardiac imaging has evolved following technological developments. In nuclear medicine, the advent of Anger camera and the use of ${ }^{99 \mathrm{~m}} \mathrm{Tc}$ labeled compounds led to implement cardiac blood-pool imaging, a gold standard technique for the assessment of biventricular function, till the validation of computed tomography and cardiac magnetic resonance (cMRI). ${ }^{3}$ Another revolution started in the 1970s with the diffusion of echocardiography: the investigation of cardiac pump

Reprint requests: Assuero Giorgetti, MD, Fondazione Toscana Gabriele Monasterio, Via G. Moruzzi 1, 56124, Pisa, Italy; asso@ftgm.it J Nucl Cardiol 2020;27:2027-30.

1071-3581/\$34.00

Copyright (C) 2018 American Society of Nuclear Cardiology. function became relatively simple, immediate and tightly linked to the cardiologists' world, with the significant limitation of a rather subjective, operator-dependent assessment. Perhaps, the success of cardiac ultrasound methodology has then forced nuclear cardiologists toward the nobody's land of myocardial perfusion, with the scope of simultaneous assessment of myocardial perfusion/function. The dream has become reality at the end of the 1990s, with the work of Germano, Bermann and other researchers: the development of gated-SPECT, permitting the noninvasive evaluation of myocardial perfusion, viability and function in the same examination, regenerated nuclear cardiology. ${ }^{4}$ Since its implementation, gated-SPECT has promised obvious advantages over perfusion evaluation only. The simple visualization of wall motion and thickening has greatly improved the identification of attenuation artefacts, with a significant increase in specificity (and overall diagnostic accuracy) of myocardial perfusion SPECT. ${ }^{5}$ The possibility to investigate regional wall motion abnormalities furnished an incremental value in the identification of coronary artery disease and for the evaluation of its extent and severity. ${ }^{6}$ Measurements of left ventricular ejection fraction (LVEF) and volumes improved prognostic stratification. ${ }^{7}$ Last but not least, the measures derived were obtained by means of semiautomatic operator-independent algorithms, resulting in a high intra- and inter-observer reproducibility. However, validation studies performed against the gold standard cMRI showed significant differences in both LVEF and volume measures, with a tendency to underestimate $\mathrm{EF}$ values and end-diastolic volumes (particularly in patients with advanced heart failure) and to overestimate endsystolic volumes (particularly in small -femalehearts). ${ }^{8,9}$ These findings recognize a complex explanation, including count statistics, spatial and temporal resolution, differing between gated-SPECT and cMRI. Moreover, cMRI allows the inclusion of the outflow tract, which is not a part of LV volume acquisition with gated-SPECT, because of the absence of counts in this 
A



B

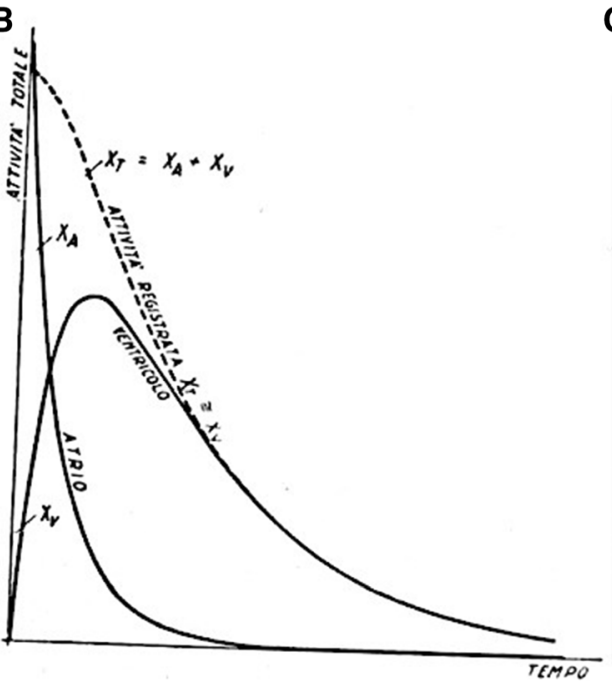

C

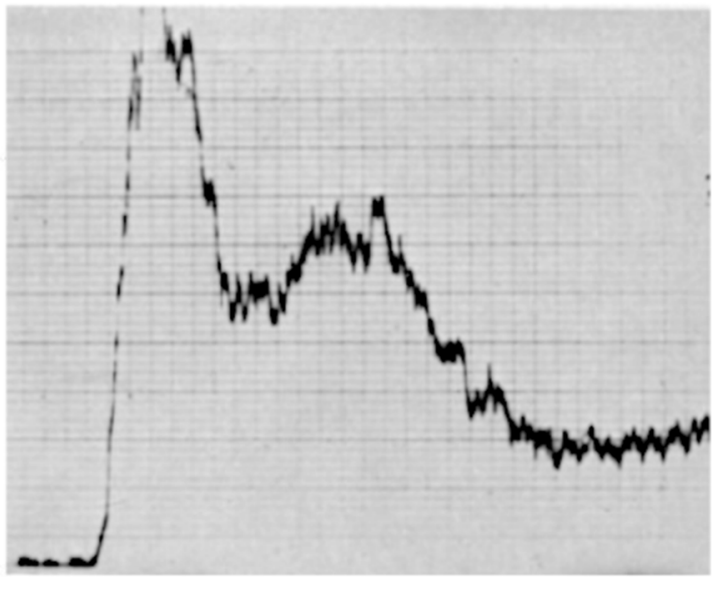

Figure 1. A Professor Luigi Donato and a co-worker preparing the patient for a radiocardiogram examination. B Graphical theory of radiocardiogram with time activity curves. C Radiocardiogram obtained in a patient with mitral stenosis: note the lengthening of the left ventricle filling time (right part of the curve).

specific area. Recently, further technological developments have introduced digital imaging in nuclear cardiology. Semiconductor cadmium zinc telluride detectors ensure a striking improvement in spatial and energy resolution, associated with a dramatic increase of count statistics, a very important issue when using a technique like gated-SPECT for evaluating LV function, strongly dependent on counts detected in each phase of the cardiac cycle. Preliminary validations of CZT cameras vs cMRI have provided encouraging results, using either a standard dose ${ }^{10}$ or a low-dose acquisition protocol. ${ }^{11}$ In the present number of the Journal, Plateau and colleagues ${ }^{12}$ compared the relative performance of the available software packages for nuclear cardiology $\mathrm{LV}$ function assessment versus the gold standard cMRI. This is not a trivial problem when making quantitative measurements of a physiological parameter. Semiautomatic software packages guarantee reproducibility but, 
being based on different mathematical and geometric approaches, can result in increased variability of the obtained measures. Authors concluded that the 4 investigated software packages provided satisfactory results, when compared to cMRI, but with wide limits of agreement.

These findings, still not surprising, strengthen the belief that $\mathrm{LV}$ function measures obtained with different techniques recognize an intrinsic validity, relative to different reference limits, and are not interchangeable. The $\mathrm{EF}$ estimation as the ratio between stroke volume and end-diastolic volume hide various pitfalls that could explain the measure heterogeneity. Techniques which are not based on the identification of myocardial borders, like cardiac bloodpool (counts based) or strain imaging (based on the assessment of average deformation), have a high reproducibility, but are not free by limitations (e.g., the counts statistics and the image quality). The other methods based on myocardial border tracing are heavily dependent on contrast resolution of the underlying algorithm. As a consequence, determination of volumes is an important source of variability in the measures obtained, as it is the case for the intracardiac contrast agents approaches against methods not using these compounds. Moreover, physiological changes can be an important source of error in the evaluation of cardiac function. EF estimate is dramatically influenced by cardiac load, and this fact implies that it should not be interpreted simply as a result of contractility without information about cardiac preload and afterload. Heart rate, too, can influence EF: in the presence of bradycardia, stroke volume increases, determining an overestimation of $\mathrm{EF}$ and myocardial function, while tachycardia can result in an underestimation. As a matter of fact, none of the techniques available up-todate takes into account the impact determined by variations of relevant physiological parameters, and when technique comparison is usually performed, no correction for these confounders is usually attempted. All in all, it is not surprising that different techniques using different physical measures furnish close but not identical results, investigating the same phenomenon, and in the dynamic evaluation of individual patients' phenotypes should not be mixed. Nevertheless, data obtained with different techniques are integral part of many cardiologic guidelines. It is conceivable that the heterogeneity in the EF and volume values estimated by different methods have a limited clinical impact either in patients with a normal cardiac function or in patients with advanced heart failure and severely depressed LV systolic function. Conversely, these pitfalls could be an important issue in heart failure with mid-range $\mathrm{EF}$, when the degree of left ventricle dysfunction is mild (i.e., EF 40-50\%). Cardiologists should therefore be very careful in the choice of the method, as well as in the management of the obtained parameters during the patients' follow-up. The study of Plateau indirectly underlines that high-quality imaging, meaning coherence in image interpretation, and a high diagnostic accuracy are crucial prerequisites in answering clinical questions such as the assessment of $\mathrm{LV}$ function. In an era of diminishing resources for clinical imaging, physician tends to use the less expensive and mostly available technique. Echocardiography has a lot of obvious advantages over the competing imaging techniques. However, not infrequently, LVEF is estimated by visual interpretation of echocardiograms, depending on the operator personal expertise and increasing the inter-observer variability. cMRI is a top player of cardiac imaging, but the scarce availability of MRI machines makes it difficult to satisfy the ever-increasing clinical demand. As demonstrated in the Plateau's paper, CZT gatedSPECT can be a valid and robust alternative to the estimation of LVEF and LV volumes and is highly reproducible and not so "ionizating" if using a lowdose protocol. On the other side, nuclear medicine specialists must maintain a continuous learning curve, not only linked to the belonging specialist branch. Parameters of myocardial function are not simply "numbers" provided by a device and a software, but require a coherent interpretation in function of the clinical question.

Since Donato's and Braunwald's first experiences, cardiology has obviously evolved. Still, LVEF and volumes are considered the most important parameters of cardiac function used by clinicians for the diagnosis of heart failure, for risk stratification, and for therapeutic decision-making.

We are all children of the lesser God radiocardiogram.

\section{Disclosure}

The authors have nothing to declare.

\section{References}

1. Donato L. Basic concepts of radiocardiography. Semin Nucl Med 1973;3:111-3.

2. Folse R, Braunwald E. Determination of fraction of left ventricular volume ejected per beat and of ventricular end-diastolic and residual volumes. Experimental and clinical observations with a precordial dilution technic. Circulation 1962;25:674-85.

3. Strauss HW, Zaret BL, Hurley PJ, Natarajan TK, Pitt B. A scintigraphic method for measuring left ventricular ejection fraction in man without cardiac catheterization. Am J Cardiol 1971;28:575-80. 
4. Germano G, Berman DS. Clinical gated cardiac SPECT. Armonk (NY): Futura Publishing; 1999. p. 387.

5. Fleischmann S, Koepfli P, Namdar M, Wyss CA, Jenni R, Kaufmann PA. Gated $99 \mathrm{mTc}$-tetrofosmin SPECT for discriminating infarct from artifact in fixed myocardial perfusion defects. J Nucl Med 2004;45:754-75.

6. Sharir T, Bacher-Stier C, Dhar S, Lewin HC, Miranda R, Friedman JD, et al. Identification of severe and extensive coronary artery disease by postexercise regional wall motion abnormalities in Tc-99m sestamibi gated single-photon emission computed tomography. Am J Cardiol 2000;86:1171-5.

7. Sharir T, Germano G, Kang X, Lewin HC, Miranda R, Cohen I, et al. Prediction of myocardial infarction versus cardiac death by gated myocardial perfusion SPECT: risk stratification by the amount of stress-induced ischemia and the poststress ejection fraction. J Nucl Med 2001;42:831-7.

8. Bavelaar-Croon CDL, Kayser HWM, van der Wall EE, de Roos A, Dibbets-Schneider P, Pauwels EK, Germano G, Atsma DE. Left ventricular function: Correlation of quantitative gated SPECT and MR imaging over a wide range of values. Radiology 2000;217:572-5.
9. Lipke CSA, Kühl HP, Nowak B, Kaiser HJ, Reinartz P, Buell U, Schaefer WM. Validation of 4D-MSPECT and QGS for quantification of left ventricular volumes and ejection fraction from gated Tc-MIBI SPET: Comparison with cardiac magnetic resonance imaging. Eur J Nucl Med Mol Imaging 2004;31: 482-90.

10. Cochet H, Bullier E, Gerbaud E, Durieux M, Godbert Y, Lederlin $\mathrm{M}$, et al. Absolute quantification of left ventricular global and regional function at nuclear MPI using ultrafast CZT SPECT: initial validation versus cardiac MR. J Nucl Med 2013;54: 556-63.

11. Giorgetti A, Masci PG, Marras G, Rustamova YK, Gimelli A, Genovesi D, et al. Gated SPECT evaluation of left ventricular function using a CZT camera and a fast low-dose clinical protocol: comparison to cardiac magnetic resonance. Eur J Nucl Med Mol Imaging 2013;40:1869-75.

12. Plateau A, Bouvet C, Merlin C, Pereira B, Barres B, Clerfond G et al. Assessment of 4 different cardiac software for evaluation of LVEF with CZT-SPECT versus CMR in 48 patients with recent STEMI. JNC-18-079-OA.R1. 